Nigerian Journal of Technology (NIJOTECH)

Vol. 36, No. 3, July 2017, pp. $806-\mathbf{8 1 3}$

Copyright@ Faculty of Engineering, University of Nigeria, Nsukka

Print ISSN: 0331-8443, Electronic ISSN: 2467-8821

www.nijotech.com

http://dx.doi.org/10.4314/njt.v36i3.21

\title{
MODELING, OPTIMISATION AND ANALYSIS OF RE-ENTRANT FLOWSHOP JOB SCHEDULING WITH FUZZY PROCESSING TIMES
}

\author{
L. Ogunwolu ${ }^{1}{ }^{*}$, A. Sosimi ${ }^{2}$ and S. Obialo ${ }^{3}$

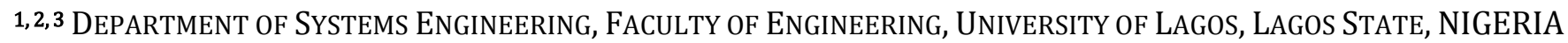 \\ E-mail addresses: ${ }^{1}$ fogunwolu@unilag.edu.ng, ${ }^{2}$ azeezadeyanju@gmail.com, ${ }^{3}$ susanobialo@gmail.com
}

\begin{abstract}
This paper presents a makespan minimization of $n$-jobs $m$-machines re-entrant flow shop scheduling problem (RFSP) under fuzzy uncertainties using Genetic Algorithm. The RFSP objective is formulated as a mathematical programme constrained by number of jobs and resources availability with traditional scheduling policies of First Come First Serve (FCFS) and the First Buffer First Serve (FBFS). Jobs processing times were specified by fuzzy numbers and modelled using triangular membership function representations. The modified centroid defuzzification technique was used at different alpha-cuts to obtain fuzzy processing times (FPT) of jobs to explore the importance of uncertainty. The traditional GA schemes and operators were used together with roulette wheel algorithm without elitism in the selection process based on job fuzzy completion times. A test problem of five jobs with specified Job Processing and Transit Times between service centres, Job Start Times and Job Due times was posed. Results obtained using the deterministic and fuzzy processing times were compared for the two different scheduling policies, FCFS and FBFS. The deterministic optimal makespan for FBFS schedule was $61.2 \%$ in excess of the FCFS policy schedule. The results also show that schedules with fuzzy uncertainty processing times provides shorter makespans than those for deterministic processing times and those under FCFS performing better than those under FBFS policy for early jobs while on the long run the FBFS policy performs better. The results underscore the need to take account of comprehensive fuzzy uncertainties in job processing times as a trade-off between time and costs influenced by production makespan.
\end{abstract}

Keywords: Fuzzy, Genetic Algorithm, Flowshop, Makespan, Processing times, Re-entrant, Schedules,

\section{INTRODUCTION}

The design of today's manufacturing and assembly facilities, is classified according to the job processing order or route. The re-entrant flow-shop has recently attracted attention (Kumar and Singhal [10]). It is a system of $m$ machines and $n$ jobs for which some or all of the $\mathrm{n}$ jobs visit some or all of the m machines more than once. The principal directions of most Re-Entrant Flow Shop Problem (RFSP) solving are the optimization of makespan, average flow time, setup cost and idle time. To meet these objectives, dispatching rules such as Shortest Processing Time (SPT), First Come First Served (FCFS), First Buffer First Served (FBFS), Last Come First Served (LCFS), Earliest Due Date (EDD), Least Slack (LS), Last Buffer First Served (LBFS) and Critical Ratio (CR) are employed. A typical example of such a re-entrant production system is the production of the semiconductor. In semiconductor manufacturing, wafers traverse flow lines several times to produce the different layers composing each circuit. Much as optimal performance of re-entrant flow-shop schedule are desirable, scarcity of resources and the uncertain nature of data hamper the achievement of such feat. In particular, the uncertain nature of data greatly influences what the outcomes of the optimization model for the prediction of optimal decisions are. In solving RFSP, several methodical approaches had been exploited.

Graves et al [6] proposed and developed a cyclic scheduling method that takes advantage of the flow character of re-entrant flow shops. In an obvious attempt to improve the same work, Wang and Choi [17] considered a chain re-entrant flow-shop that minimize makespan using branch and bound optimization algorithm and three approximation algorithms with worst-case performance guarantee. Chen et al [3] applied hybrid tabu search (HTS) to minimize the makespan of jobs in re-entrant flow-shops. Lee and Lin [11] proposed a simulated genetic algorithm model for scheduling reentrant flow shops, the work amply demonstrated the potential of soft computing techniques to RFSP and near optimal solution and in some cases an optimal solution can be obtained. Dugardin et al [4] worked on hybrid

\footnotetext{
* Corresponding author, tel: +234-803-353-8328
} 
multi-objective methods to solve re-entrant flow shops with two objectives. Like previous work, no attempt was made at scheduling under any form of uncertainty.

Uncertainties are unpredictable events that disturbs the operations in a manufacturing system (Wang, et al. [18]). These are in two broad categories (Gholami and Zandieh [5]; Ouelhadj and Petrovic [13])

i. Resource-related uncertainty: machine breakdown, unavailability or failure of tools, loading limits, and defective materials (materials with the wrong specifications), etc.

ii. Job-related uncertainty: job cancellation, due-date changes, early or late arrival of jobs, changes in job priority, and changes in job processing time, etc.

There are a number of uncertainties inherent in measurement and specification of parameters and variables in shop scheduling. These include uncertainties in processing times, set-up time, due date and costs. They can be due to incomplete knowledge or uncertain environment. Generally, uncertain data can be expressed by probabilistic functions or fuzzy sets. Fuzzy sets provide an appropriate tool for handling imprecise information (Wang, et al [18]).

Hapke and Slowinski [7] noted that fuzzy sets and logic can be used to tackle uncertainties inherent in actual flow shop scheduling problems. Tsujimura, et al [16] showed that fuzzy set theory can be useful in modeling and solving flow shop scheduling problems with uncertain processing times. In their work, the satisfaction degrees for job completion times are described using fuzzy sets, and the objective is to obtain a job sequence whose completion time has maximum degree of satisfaction. Ishibuchi, et al [9] used triangular, trapezoidal and bell-shaped membership functions to describe jobs data. Chanas and Kasperski [2] minimized lateness in a single machine scheduling problem with fuzzy processing times and fuzzy due dates. Puente, et al [15] presented a job shop in which uncertain duration was modeled using triangular fuzzy numbers and solved by a fast local search. Yao and Lin [19] reported that fuzzy flow-shop model is an extension of the crisp flowshop problem. RFPS under uncertainties is an emerging research area and methodologies for scheduling under uncertainty are aimed at producing feasible, robust and optimal schedules. Li and Ierapetritou [12] and Huang and Fujimira [8] provide an effective fuzzy based multicriteria genetic algorithm to solve re-entrant flow shop scheduling with the objective of minimizing the total turnaround time. Applying fuzzy set theory to scheduling optimization has primarily focused on use of metaheuristic techniques to obtain near-optimal solutions. This paper in consonance investigates the effects of uncertain processing times described by triangular fuzzy sets for the fuzzy processing times in RFSP.

\section{THE RE-ENTRANT FLOWSHOP PROBLEM DESCRIPTION}

A typical configuration of a RFSP is illustrated in Figure 1. It consists of buffers $b_{k}, k=1,2, \ldots, K$ used to hold jobs, $J_{j}, j=1,2, \ldots, J$ in queue for processing at different service centres $N_{i}, i=1,2, \ldots, n$ which have processing times $T_{k}$ representing regimes of jobs through buffers $k=1,2, \ldots, K$.

The layout consists of an open re-entrant line with service centers $N_{i}$ and finite capacity storage buffers $b_{k}$. Once a job part $J_{j}$ enters the line through buffer $b_{k}$, it is processed by the machines in the corresponding service center. It follows the process through buffer $b_{k}$ and through the re-entrant lines to next buffer. The buffer through which a job enters the service center distinguishes the operation to be carried out and the processing time that is allocated to that operation. The sequence of operation for each job is $b_{1}, b_{2}, \ldots, b_{k}, \ldots, b_{L}$. The objective is to obtain a job sequence that minimizes makespan. In the ensuing Mathematical model developed, the transit time between the service centre housing buffer $k$ and the next is $V_{k, k+1}$; the due date for jobs is represented as $d_{j}, j=1,2, \ldots, \mathrm{J}$; the job completion times for individual jobs are $C_{j}, j=1,2, \ldots, J$ $; C_{\max }$ is the maximum completion time for a set of jobs while $t_{i j k}$ represents the start time of job $j$, at service centre $i$, through buffer $k$.

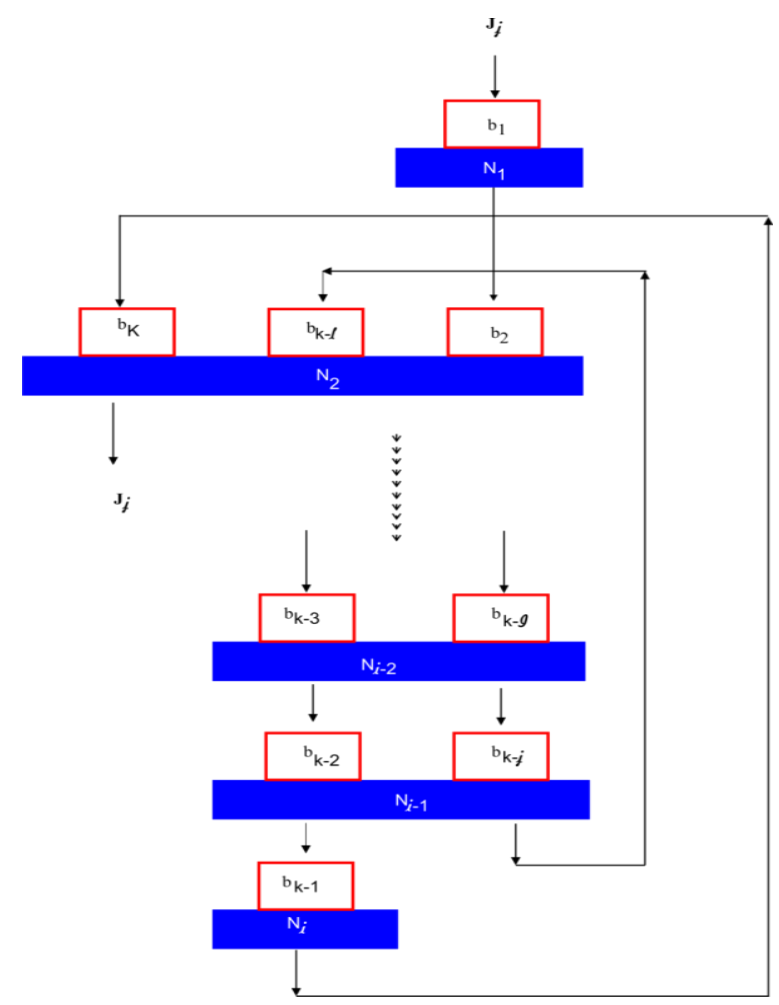

Figure 1: A Typical Reentrant Flowshop Generalized Configuration 


\subsection{Scheduling Policies at Service Centres}

The choice of jobs to process in each service centers are often dependent on scheduling policies. The following scheduling policies were considered in the modeling process.

\subsubsection{First Buffer First Served (FBFS)}

Jobs are processed in the sequence in which they entered the buffers according to the given buffer ranking. An idle machine at processing centre $i$, with $n_{i}$ buffers processes the first job in buffer $b_{k}$, where $b_{k} \in\left\{b_{1}, b_{2} \ldots, b_{L}\right\}$ the set of buffers in the system and $b_{k} \in\left\{b_{k}\right\}_{i}$ the set of buffers attached to processing centre i. Where $b_{h}, b_{j} \in\left\{b_{k}\right\}_{i}$ contain jobs to process, the job at the buffer $b_{h}$ which comes ahead of buffer $b_{j}$ in the serial ranking of the system buffers, $\left\{b_{1}, b_{2} \ldots, b_{h}, \ldots, b_{j}, \ldots b_{L}\right\}$ is processed first ahead of the one at $b_{j}$.

\subsubsection{First Come First Served (FCFS)}

Jobs are processed in the sequence in which they entered the shop. An idle machine at processing centre $i$, processes the waiting job that arrived the earliest, regardless of which buffer $b_{k} \in\left\{b_{k}\right\}_{i}$ it is in.

\subsection{The Mathematical Model}

The objective herein is to obtain a job sequence that minimizes makespan and gives the start times of individual jobs at the service centers based on the following assumptions:

i. The system under consideration is an open reentrant line.

ii. The machines in each service center are identical.

iii. The machines can process only one part at a time.

iv. Any two consecutive operations of a job must be processed on different machines.

v. Every job may visit certain service centers more than once.

vi. All jobs are ready for processing at time zero at which the machines in the service centers are idle and immediately available for work.

vii. Preemptive scheduling: no operation may be interrupted when it has already been started.

viii. Once an operation is started, it must be completed before another one can be started on that machine.

ix. Machines never break down and are available throughout the scheduling period.

$\mathrm{x}$. Unlimited storage or buffer capacities in between successive machines (no blocking).

xi. A job has to be processed at each service center on only one of the machines (no parallel machines).

The RFSP proposed in this paper was modelled as $0-1$ integer linear programming using pre-defined parameters and variable representations. Using $T_{k}$ to represent the processing time required for a job at the stage for which buffer $k$ is attached to a service centre and $t_{i j k}$, the scheduled start time of job $j$, at service centre $i$, to which a buffer $k$ is attached, the Mathematical Programming model is:

$$
\text { Minimize } \sum_{i=1}^{I} \sum_{j=1}^{J} \sum_{k=1}^{K}\left(t_{i j k}+T_{k}\right)
$$

Subject to:

$$
\begin{aligned}
& \text { (C01) } \quad t_{111}=0 \\
& (\mathrm{C} 02) \quad t_{i j k}+T_{k}+V_{k, k+1} \leq t_{i+1, j, k+1} \\
& \forall i=1,2, \ldots, I ; j=1,2, \ldots, J ; k=1,2, \ldots, K \\
& (\mathrm{C} 03) \quad t_{i j v} \leq t_{i j+1, w} \forall v, w \in K ; k=1,2, \ldots, K \\
& (\mathrm{C} 04) \\
& \quad Y\left(t_{i r w}-t_{i q v}\right)<0
\end{aligned}
$$

$$
Y=\left\{\begin{array}{cc}
1, & \text { if buffer } w<v \\
0, & \text { otherwise }
\end{array} \quad r \neq q ; v, w \in\{k\} ; r, q \in\{j\}\right.
$$

\subsection{Problem Constraints}

Constraint (1) set stipulates that jobs are ready for processing i.e. start time for the first job at the first service center through the first buffer. Constraint set (2) ensures that the operation on job, $j$, through a buffer, $k$, in a service center, $i$, is completed before next operation on the job starts in the succeeding service center, $i+1$, through buffer $k+1$. Constraint set (3) stipulates jobs, $j$, are scheduled sequentially in a particular service center, $i$, according to the order in which they come into the system irrespective of the buffer they arrive to. Set of Constraints (4) restricts the model to schedule jobs according to the FBFS buffer preference.

Constraint sets (1), (2) and (3) are used for the First Come First Served (FCFS) scheduling policy, while sets (1), (2), (4) and the binary variable $Y$ in Constraint set (5) are used for the First Buffer First Served (FBFS) scheduling policy.

With respect to the incorporation of fuzzy uncertainty in processing times, the various processing times, $t_{i j k}$ for processing at service centre $i$, of job $j$, through buffer $k$, are simply changed to $\left(\overline{t_{i j k}}\right)_{\alpha}$ representing the fuzzy processing time at service centre $i$, of job $j$, through buffer $k$, at an alpha-cut of $\alpha, 0 \leq \alpha \leq 1$.

\section{DERIVATION OF FUZZY INPUT MODEL}

In other to capture uncertainties of jobs processing time, fuzzy set principle was utilized and job processing time $\overline{\boldsymbol{T}}_{\boldsymbol{k}}$ are modelled by triangular fuzzy number (TFN). The fuzzy parameters are specified by the triplet $[a, b, c]$ as depicted in Figure 2. The membership value of the $\boldsymbol{T}$ is denoted by $\mu(\boldsymbol{T}), \quad \boldsymbol{T} \in \mathcal{R}$. The fuzzy parameters $\left(a_{\alpha}, b, c_{\alpha}\right)$ obtained at a membership grade of $\alpha \in[0,1]$ is called the alpha-cut of the set, modified centroid defuzzification technique in [14] was used at different alpha-cut ranging from 0 to 1 at intervals of 0.1 to derive the fuzzy processing time $\overline{\boldsymbol{T}}_{\boldsymbol{k}}$ as illustrated in Eqn. 6 . 


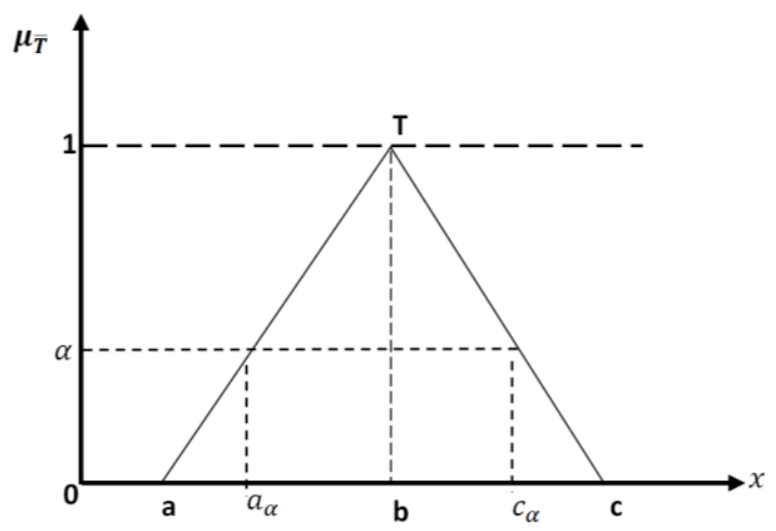

Figure 2: Triangular Membership Function of Fuzzy Numbers

$$
\bar{T}_{k}=\left[t_{c}-\left(t_{c}-t_{b}\right)\left(t_{c}-t_{a}\right)^{1 / 2}\right]
$$

Here $t_{a}, t_{b}$ and $t_{c}$ are lower bound, mean membership value or a modal point and upper bound respectively of the relevant processing times [1]. A principal objective of RFSP is to determine the optimum schedule, as a result in this study, the feasible schedules under fuzzy uncertainties are also obtained and compared so as to identify optimal schedule.

\section{TEST PROBLEM, SOLUTION METHODOLOGY, RESULTS AND ANALYSIS}

\subsection{The Test Problem}

The test problem consists of 3 Service stations and 5 buffers as depicted in Figure 3. A test problem was constituted to validate the developed model whose generalized form was enunciated on in the last section. The test problem has 5 buffers $(K=5)$, three service centres $(\mathrm{I}=3)$ and five jobs available for processing $(\mathrm{J}=$ 5). Tables 1,2 and 3 below specify the Job Processing and Transit Times, Job Start Times and Job Due times respectively.

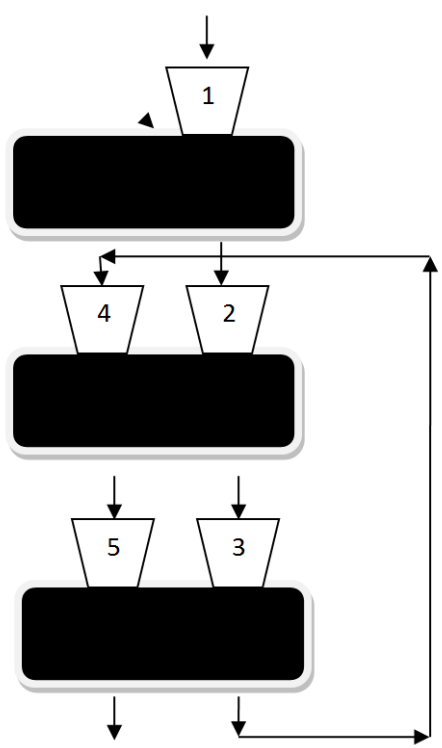

Figure 3: Configuration of the Test Problem for RFS Model

Table 1: Job Processing (Deterministic and Fuzzy) and Transit Times

\begin{tabular}{ccccc}
\hline \multicolumn{2}{l}{ Job Processing Times $\left.\left(T_{k} / \bar{T}_{k}\right)\right)$ in hours } & \multicolumn{2}{c}{ Job Transit Times $\left(V_{k, k+1}\right)$ in hours } \\
\hline$T_{k}$ & Deterministic & Fuzzy & $V_{k, k+1}$ & Value \\
$T_{1}$ & 2 & $(1.05,2,3.64)$ & $V_{0,1}$ & 0 \\
$T_{2}$ & 3 & $(1.57,3,4.22)$ & $V_{1,2}$ & 0.1 \\
$T_{3}$ & 2 & $(1.89,2,3.13)$ & $V_{2,3}$ & 0.4 \\
$T_{4}$ & 0.5 & $(0.08,0.5,2.75)$ & $V_{3,4}$ & 0.4 \\
$T_{5}$ & 0.8 & $(0.24,0.8,1.9)$ & $V_{4,5}$ & 0.1 \\
\hline
\end{tabular}

Table 2: Job Start Times (hours)

\begin{tabular}{|c|c|c|c|c|c|c|}
\hline \multicolumn{3}{|c|}{ Known Start Times (hours) } & \multirow{2}{*}{\multicolumn{4}{|c|}{ Unknown Start Times (Decision Variables), $t_{i j k}$}} \\
\hline Job No, j & $t_{i j k}$ & Value & & & & \\
\hline 1 & $t_{111}$ & 0 & $t_{212}$ & $t_{313}$ & $t_{214}$ & $t_{315}$ \\
\hline 2 & $t_{121}$ & 8 & $t_{222}$ & $t_{323}$ & $t_{224}$ & $t_{325}$ \\
\hline 3 & $t_{131}$ & 12 & $t_{232}$ & $t_{333}$ & $t_{234}$ & $t_{335}$ \\
\hline 4 & $t_{141}$ & 15 & $t_{242}$ & $t_{343}$ & $t_{244}$ & $t_{345}$ \\
\hline 5 & $t_{151}$ & 19 & $t_{252}$ & $t_{353}$ & $t_{254}$ & $t_{355}$ \\
\hline
\end{tabular}

Table 3: Job Due Times (hours)

\begin{tabular}{lllll}
\hline Job number j & 1 & 2 & 3 & 4 \\
\hline Due time (hours) & 20 & 23 & 19 & 24 \\
\hline
\end{tabular}




\subsection{Model Solution Methodology}

The re-entrant flow-shop problem was solved using generic Genetic Algorithm (GA) as described in Algorithm 1 below.

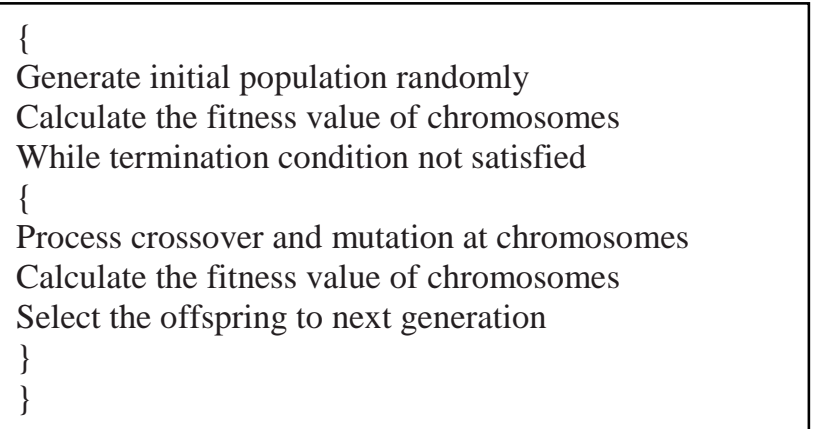

Algorithm 1: Generic Genetic algorithm.

\subsubsection{The Genetic Algorithm Parameters}

Genetic Algorithm chromosomes are constituted as bytestring lengths for each $t_{i j k}$, of service station i $(i=2,3)$, job j $(j=1,2, \ldots, 5)$, buffer $\mathrm{k}(k=1,2, \ldots, 5)$, of the 20 decision variables denoting the scheduled starting times of jobs (Table 2). Thus each chromosome has a length of 100 bytes.

A constant population size of 200 chromosomes was adopted with a 2-point crossover, crossover-probability of 0.8 and byte-wise mutation probability of 0.2 . Selection was by roulette wheel mechanism without elitism.

\subsubsection{The Fitness Function}

The fitness function was constructed in line with the objective of the problem to minimize the makespan of jobs schedule. Thus, for each chromosome the fitness is evaluated as the reciprocal of the sum of the start time and processing times $t_{i j k}+T_{k}$ over all jobs, service stations and buffers, so that the minimum makespan can be returned as optimum subject to the constraint for any particular scheduling policy.

\subsubsection{Termination Criterion}

The genetic algorithm execution was terminated when either the upper limit of generations (200 generations in this respect) or the maximum number of individual chromosomes (the population size) converge on a minimum fitness value whichever came first. The individual chromosome with the lowest value of the fitness function represents the solution returned by this algorithm. This individual represents the production schedule with minimum makespan.

\subsubsection{Execution of the Algorithm}

The test problem was run with MATLAB optimization toolbox. All the toolbox functions are MATLAB M-files made up of MATLAB statements that implement GA. The hardware used for running the $\mathrm{M}$-file programme is an All-In-One Intel Pentium CPU G2020 @ $2.90 \mathrm{GHz}$ with 4.0GB Memory.

\subsection{Results and Analysis \\ 4.3.1 Deterministic Model Results}

The performance of running the test problem using FCFS and FBFS policies with deterministic processing times are presented in Figure 5. Due to buffer preferences, the FBFS policy shows earlier jobs waiting for latter jobs that are to be processed in a preferred buffer as depicted in the Gantt charts illustrated in Figures 6 and 7.

From the Gantt chart in Figures 6 and 7, more time is required to process same jobs with the FCFS policy than with FBFS policy, even though the FCFS policy out performs FBFS policy in terms of makespan. This is buttressed by the results depicted in Figures 8 and 9 .

Figure 8 shows that for some specific jobs (particularly jobs 4 and 5), FBFS performed better than FCFS. The superiority of FCFS in this instance is only for the first 2 jobs to come into the production floor (jobs 1 and 2) for which the makespan differences between the two policies are more pronounced in favour of FCFS. The two policies appear to be at par in policy performance for Job 3. Figure 9 shows that the optimal makespan returned for the FCFS (25.299 hours) is $61.2 \%$ in excess of the optimal makespan for the FBFS policy (15.694 hours). However, it is obvious from Figure 8 that the FCFS was only superior to FBFS in Total optimal makespan over all jobs.

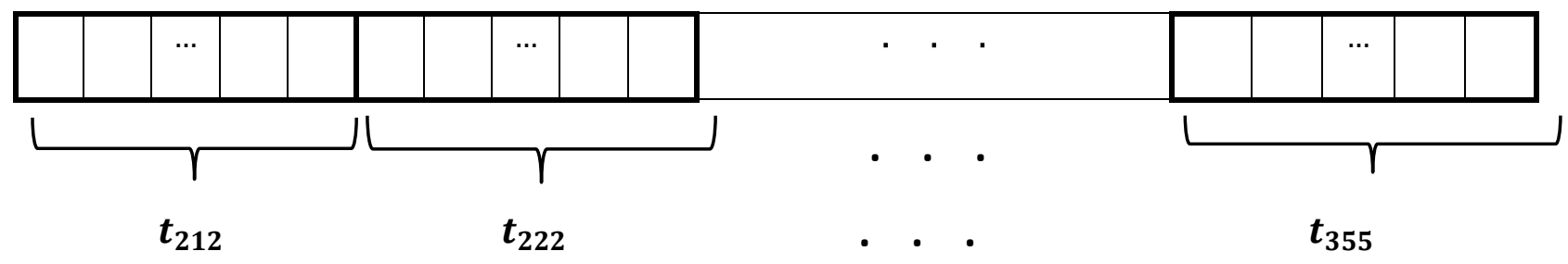

Figure 4: Representation of Solution Strings in Chromosomes 


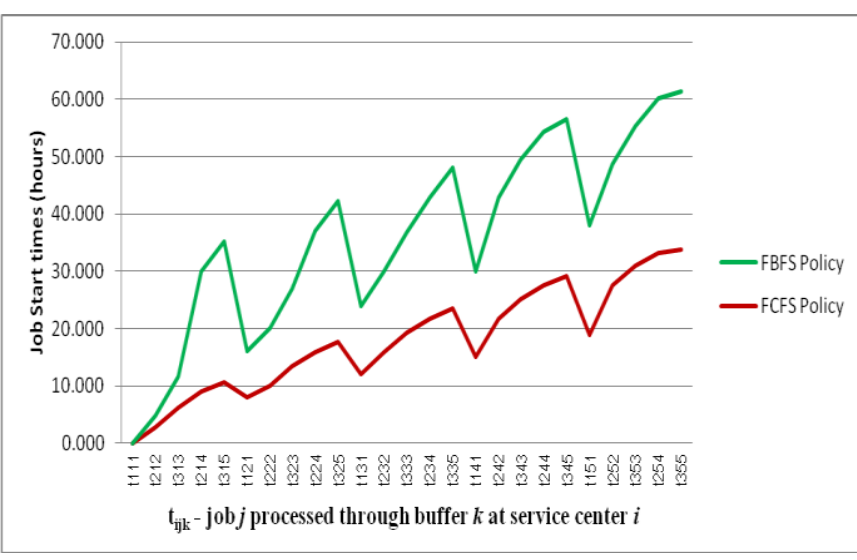

Figure 5: Deterministic Scheduled Start Times for FBFS and FCFS Scheduling Policies

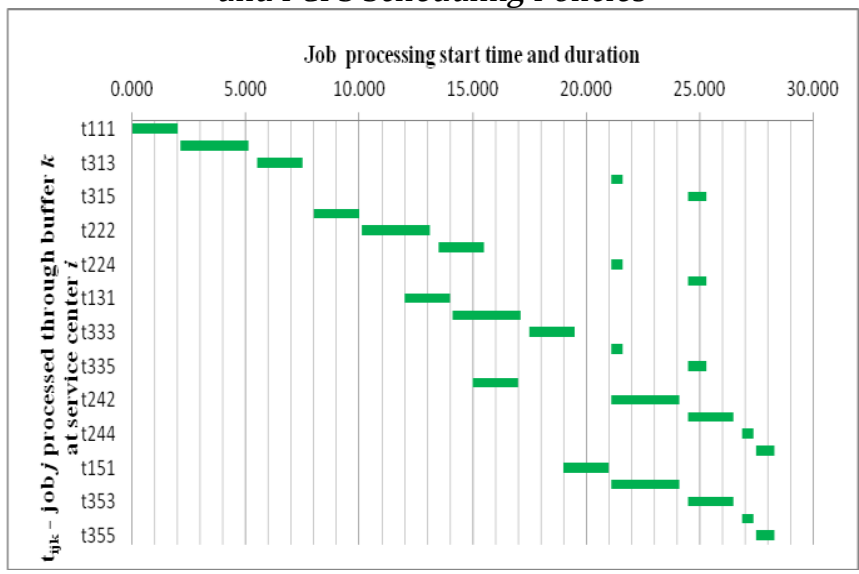

Figure 7: The Deterministic Schedule for FBFS Policy

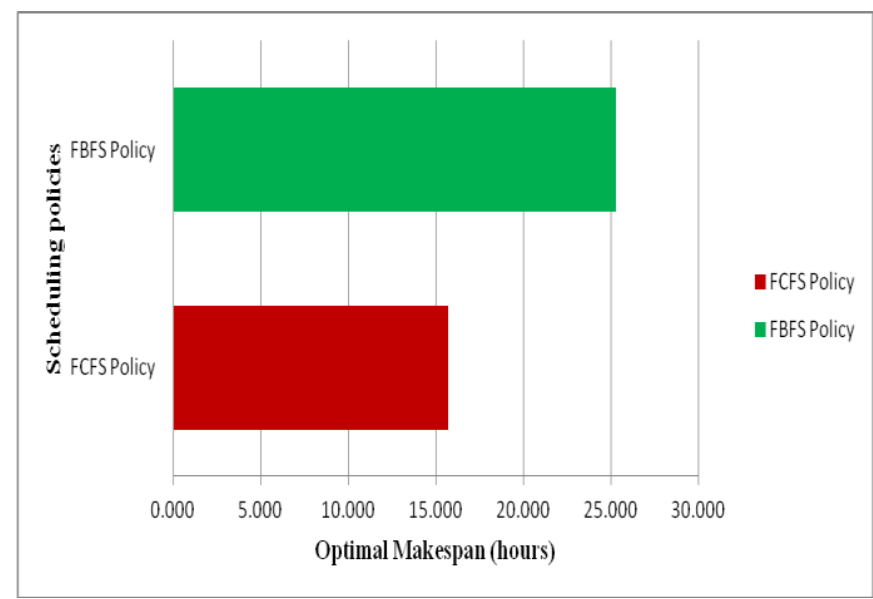

Figure 9: Maximum Optimal Job Makespan for the Deterministic Schedule

The import of this deductions and the trend is that while FCFS will prove a better policy for early jobs, as the jobs grow in number the FBFS policy will eventually prove superior at the expense of meeting due dates which FCFS ensures better.

\subsubsection{Fuzzy Model Results}

The fuzzy schemes support analysis at different degrees of fuzziness. This gives different sequences as presented in Figures 10, 11, 12 and 13. Figure 10 shows the distribution of starting times of each jobs at different

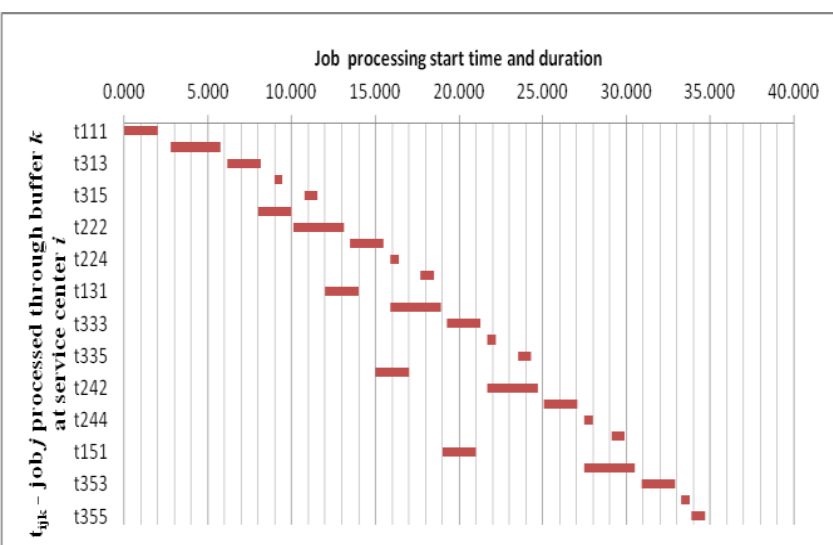

Figure 6: The Deterministic Schedule for FCFS Policy

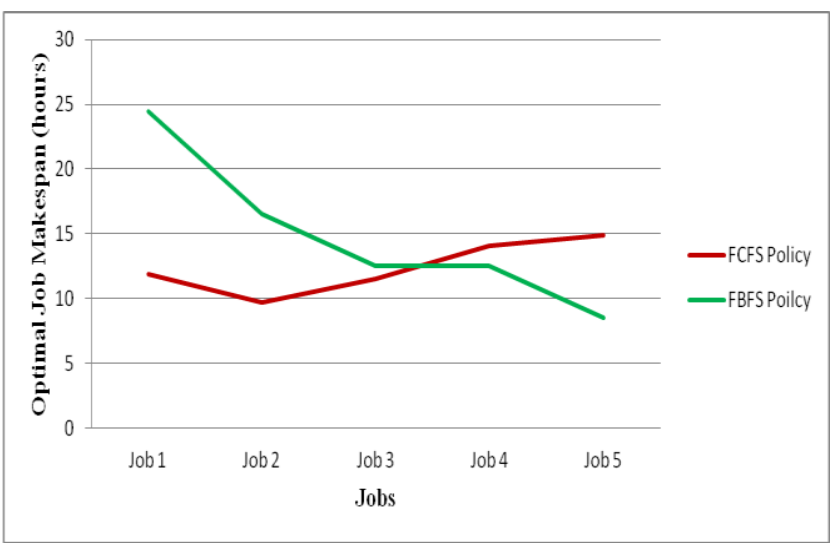

Figure 8: Optimal Makespan for the Jobs in the Deterministic Condition

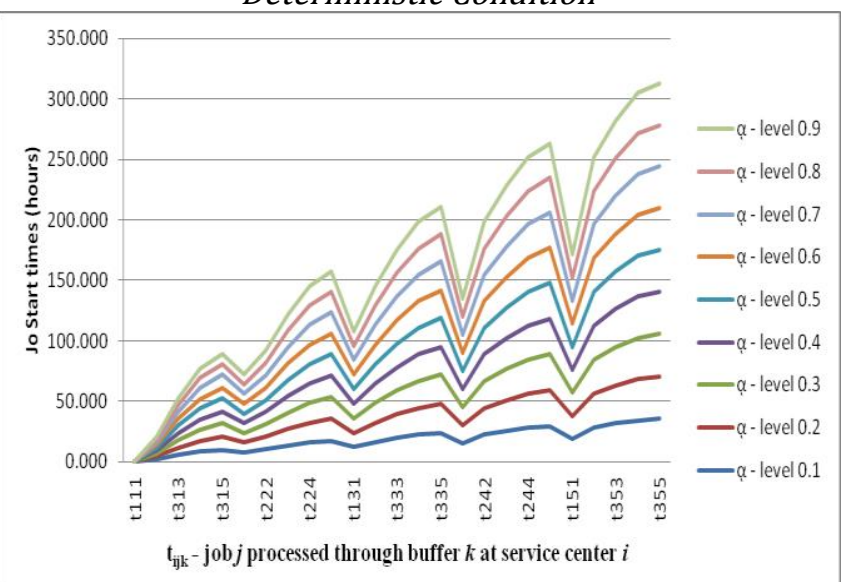

Figure 10: Scheduled Start Time for the FCFS Policy at Different $\alpha$-Levels

buffer/service station over varying degree of fuzzy $\alpha$-level of processing times.

Figure 10 shows short ridge heights of start times, rapidly growing ridge height over time and increasing dispersion of start times between different alpha-levels from buffer to buffer. This is an indication of early start times which will grow into delayed start times as jobs increase on the production floor. This is a reflection of the fact that this scheduling policy, even though an improvement on the deterministic version will result in the delay of later jobs as all jobs have to take to the FCFS 
order regardless of when the jobs have been completed at previous service centres.

Figure 11 depicts the corresponding variation of makespan with growing alpha-levels for the FCFS start time distribution discussed above. Figure 11 shows decreasing makespan for the FCFS policy as alpha grows from 0.1 to 0.9 . This shows a positive influence of FCFS as fuzzy (alpha-cut) certainty grows. By implication uncertainty can deteriorate makespan.

Figure 12 shows the distribution and variation of start times for the different jobs under FBFS policy. Unlike the counterpart distribution under the FCFS policy, Figure 12 shows taller ridge heights of start times for early jobs with gentle ridge height increase over time and fairly even dispersions of start times between different alphalevels from buffer to buffer. This is an indication that early job start times under FBFS policy may have high start times but that the policy evens out delays in the start times in later jobs which will culminate into them starting earlier than if FCFS policy has been used. This accounts for the lower terminal start time (250 hours) for FBFS compared to 300 hours for FCFS at alpha level of 0.9. This is a reflection of the fact that the FBFS policy will perform better than the FCFS policy and result in shorter makespans on the long run.

For both FCFS and FBFS policies, the schedules tend to the deterministic case while the optimal makespans decrease with increase in alpha-level. This underscores the importance of critical assessment of the level of uncertainty involved in processing times at different production floor service units so that informed scheduling decisions can be made at any time and for any job combinations.

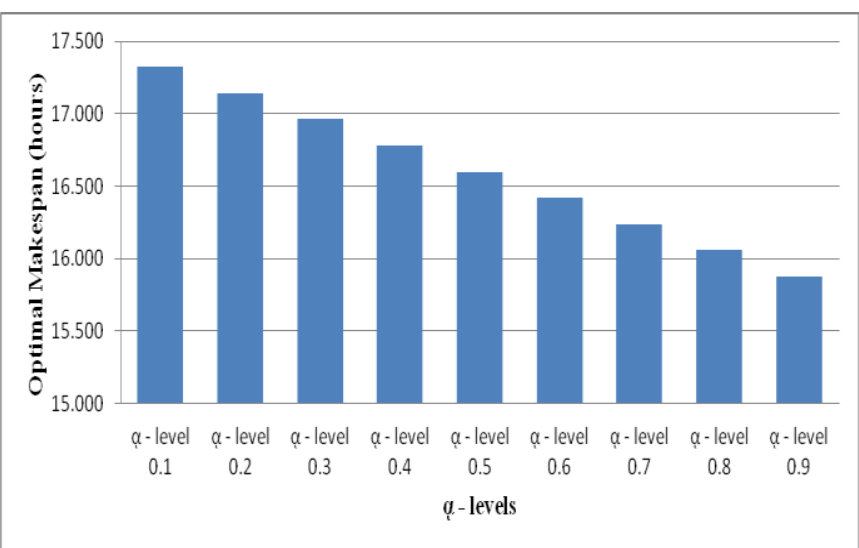

Figure 11: Makespans for the FCFS Policy at Different $\propto$ - Levels
Table 4 presents value comparisons of the Optimal Makespan over all jobs at different alpha-levels of fuzzy processing times under the two policies. From Table 4, it is obvious that variations in Optimal Makespan under the FBFS is fairly constant and thus not as well pronounced as in the case of FCFS. This may tilt the table in favour of the former policy especially where processing time uncertainties are not known or evaluated at expense of longer makespan which literarily translates to greater production costs.

Statistical test on the two streams of policy makespan results shows a high correlation of 0.997 in trend between the streams of values, attesting to similarity in trend (not in values) of the two. A one-factor Analysis of Variance (ANOVA) test conducted on the two, on the other hand, at $95 \%$ confidence level $(\alpha=0.05)$ returned an F-value of 2784.753 (and a p-value of $2.23 \times 10^{-19}$ ) compared to F-Critical of 4.4940 showing that there is a high statistical significance between the difference of the means of the two streams of values. The FCFS policy thus offer a more robust scheduling policy which can result in shorter makespans and by implication much lower production costs especially if good estimation of level of fuzzy uncertainty are handy for scheduling. However, as the number of jobs grows the FBFS policy, going by the analysis above will outperform the FCFS from some point.

In summary, a robust and cost-effective scheduling policy for any instance will be a trade-off between level of uncertainty and makespan. The choice of the $\alpha$-level will be then be determined by what is considered as optimality for the scheduler while considering the job start times and the makespan for each job.

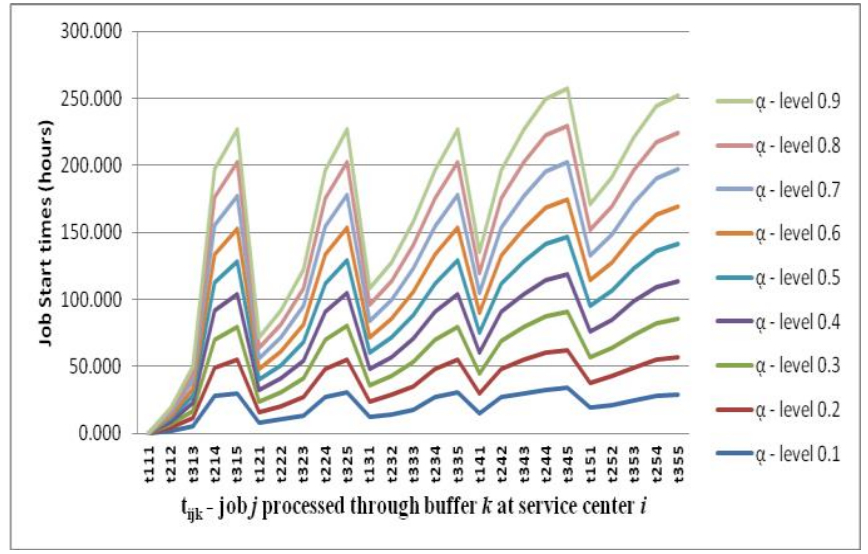

Figure 12: Scheduled Start Times for the FBFS Policy at Different $\propto-$ Levels

Table 4: Optimal Makespans at Different $\alpha$-level of Fuzzy Processing Times for FCFS and FBFS

\begin{tabular}{lccccccccc}
\hline \multicolumn{10}{c}{ Fuzzy $\alpha$ - Level } \\
\hline POLICY & 0.1 & 0.2 & 0.3 & 0.4 & 0.5 & 0.6 & 0.7 & 0.8 & 0.9 \\
FCFS & 17.326 & 17.141 & 16.961 & 16.781 & 16.595 & 16.417 & 16.239 & 16.058 & 15.874 \\
FBFS & 25.549 & 25.52 & 25.492 & 25.466 & 25.436 & 25.409 & 25.383 & 25.355 & 25.326 \\
\hline
\end{tabular}




\section{CONCLUSIONS AND POSSIBLE EXTENSIONS}

This paper has presented GA approach to RFSP with different scheduling policies under fuzzy processing time uncertainty. The proposed technique which is premised on the use of fuzzy job processing times has shown a higher prospect of results with respect to effectiveness. On comparison with the deterministic job processing time, the fuzzy model under the two scheduling policies demonstrated some level of superiority especially as displayed in the optimal makespan of the test problem. While a higher makespan time is required when scheduling with deterministic processing time for the scheduling policies considered. The FCFS performs better as against FBFS scheduling policy for early jobs but is out-performed by FBFS on the long run. Furthermore, scheduling under uncertain processing times inspires informed decisions on the choice of schedules on production flow lines.

For future works, a hybrid genetic algorithm constrained by other scheduling policies under stochastic, fuzzystochastic and stochastic-fuzzy uncertainties conditions may be used. Also, another direction of further study will be to explore the stability of system resources and production quality under both deterministic and uncertain schedule for multiple machines RFSP.

Conflict of Interest: The authors declare that there is no conflict of interest whatsoever on this article or the parent research body from where it was curled.

\section{REFERENCES}

[1] Adamopoulos G. I. and Pappis C. P.“Fuzzy Linguistic Job Scheduling". European Journal of Operations Research 92(3), 1996, 625 - 636.

[2] Chanas S. and Kasperski A. "Minimising maximum lateness in a single machine scheduling problem with fuzzy processing times and fuzzy due dates", Engineering Applications of artificial Intelligence, 14 (3), 2001,377 - 386.

[3] Chen, J. S., Pan, J. C. H., and Wu, C. K. "Minimizing makespan in reentrant flow-shops using hybrid tabu search", The International Journal of Advanced Manufacturing Technology, 34 (3-4), 2007,353 - 361.

[4] Dugardin, F., Yalaoui, F. and Amodeo, L"Hybrid MultiObjective Methods to Solve Reentrant Shops", International Journal of Applied Logistics (IJAL), 3 (4), $2012,15-32$.

[5] Gholami, M. and Zandieh, M."Integrating simulation and genetic algorithm to schedule a dynamic flexible job shop", Journal of Intelligent Manufacturing 20 (4), 2009, 481 - 498.

[6] Graves, S. C., Meal, H. C., Stefek, D., and Zeghmi, A. H. "Scheduling of re-entrant flow shops", Journal of Operations Management, 3(4), 1983,197 - 207.
[7] Hapke, M., \& Slowinski, R. Fuzzy set approach to multiobjective and multi-mode project scheduling under uncertainty, Scheduling under fuzziness, 2000, 197 221.

[8] Huang, I., \& Fujimura, S. "A fuzzy-based multi-term genetic algorithm for reentrant flow shop scheduling problem", IEEE International Conference on Industrial Engineering and Engineering Management (IEEM), 2013, pp. $305-309$.

[9] Ishibuchi, H., Murata, T., and Lee, K. H. Formulation of fuzzy flowshop scheduling problems with fuzzy processing time, Proceedings of the Fifth IEEE International Conference on Fuzzy Systems1, 1996, 199 $-205$.

[10] Kumar G. and Singhal S. "Genetic Algorithm Optimization of Flow Shop Scheduling Problem with Sequence Dependent Setup Time and Lot Splitting", International Journal of Engineering, Business and Enterprise, 2013, pp. 62 - 71.

[11] Lee, C. K. M. and Danping Lin."Hybrid genetic algorithm for bi-objective flow shop scheduling problems with reentrant jobs", IEEE International Conference on Industrial Engineering and Engineering Management (IEEM), 2010, pp.1240 - 1245.

[12] Li, Z., \& Ierapetritou, M. "Process scheduling under uncertainty: Review and challenges",Computers \& Chemical Engineering, 32(4), 2008, 715 - 727.

[13] Ouelhadj, D., \& Petrovic, S. A survey of dynamic scheduling in manufacturing systems. Journal of scheduling, 12(4), 2009, $417-431$.

[14] Ogunwolu L., Sosimi A. A, Shittu K. O., Oyetunji, E. O. and Oke, S. A. "Maintenance Job Scheduling: A Multi-Criteria Approach Under Stochastic-Fuzzy Uncertainty", KMITL Science and Technology Journal, 7(2), 2007, 77 - 91.

[15] Puente Peinador, J., Rodríguez Vela, M. d. C., \& González Rodríguez, I. "Fast local search for fuzzy job shop scheduling",Frontiers in Artificial Intelligence and Applications, 2010.

[16] Tsujimura, Y., Park, S. H., Chang, I. S., \& Gen, M. "An effective method for solving flow shop scheduling problems with fuzzy processing times", Computers \& Industrial Engineering, 25(1), 1993, 239 - 242.

[17] Wang, K., \& Choi, S. "A decomposition-based approach to flexible flow shop scheduling under machine breakdown",International Journal of Production Research, 2012, 50 (1), 215 - 234.

[18] Wang, M. Y., Sethi, S. P., and van de Velde, S. L. "Minimizing makespan in a class of reentrant shops", Operations Research, 45(5), 1997, 702 - 712.

[19] Yao, J. S., and Lin, F. T. "Constructing a fuzzy flow-shop sequencing model based on statistical data", International Journal of Approximate Reasoning, $29(3), 2002,215-234$. 